\title{
EVALUATION OF AN ONLINE FERMENTATION MONITORING SYSTEM
}

\author{
M. Gyalai-Korpos, A. Fehér, Zs. Barta* and K. Réczey \\ Department of Applied Biochemistry and Food Science, Budapest University of Technology and Economics, \\ H-1111 Budapest, Szent Gellért tér 4. Hungary
}

(Received: 28 June 2012; accepted: 12 November 2012)

\begin{abstract}
The need to introduce promising bioethanol production technologies calls for advanced laboratory techniques to study experiment designs and to obtain their results in a quick and reliable way. Real time monitoring based on general principles of ethanol fermentation, such as effluent $\mathrm{CO}_{2}$ volume, avoids time consuming steps, long lasting analyses and delivers information about the process directly. A device based on the above features and capable for real time monitoring on parallel channels was developed by the authors and is described in this paper. Both for calibration and for fermentation, test runs were carried out on different days and channels. Statistical evaluation was based on the obtained data. According to the $t$-test $(\mathrm{P}=0.05)$ and Grubbs analysis, the calibration method is reliable regardless of the date of calibration. When evaluating the fermentation results by ANCOVA acceptable standard derivations were obtained as impact of channel $(58.8 \mathrm{ml})$, date $(82.1 \mathrm{ml})$ and incorporating all impacts $(116.2 \mathrm{ml})$. The final ethanol concentrations calculated based on the gas volume were compared to ones determined by HPLC and an average difference of $10 \%$ was found. Thus, the device proved to be advantageous in monitoring fermentation.

Keywords: ethanol, fermentation, monitoring, device, $\mathrm{CO}_{2}$
\end{abstract}

Ethanolic fermentation is an enzymatic disassembly of organic matters. Bioethanol can be produced from a wide range of raw materials built up of either sugar, starch or lignocellulose. Whatever the raw material is, it must be decomposed first to simple sugars of six carbons of which ethanol can be produced by yeast strains, usually by common baker's yeast (Saccharomyces cerevisiae) with the exclusion of oxygen (anaerobic conditions). Based on the stoichiometry of fermentation, theoretically one mole of six carbon sugar delivers 2 moles of ethanol and $\mathrm{CO}_{2}$, e.g. same molar amounts of the two compounds are formed (Eq. 1). Of course, this equation describes only the quantity of hexose converted into ethanol, but some of the hexoses are either consumed by the yeast to support growth (under low oxygen conditions this is less than 5\% of the sugars) or other metabolites are also produced (glycerol, lactic and succinic acid, etc.) (Russell, 2003). Nevertheless, these other metabolites are small in quantity compared to the amount of the ethanol produced (Russell, 2003). Consequently, glucose conversion is not associated to this equation, but the theoretical ratios of the product may be useful to estimate the amount of one from the amount of the other. Moreover, in the presence of oxygen (at the beginning of the fermentation) respiration also occurs leading to excess $\mathrm{CO}_{2}$ formation. Although, these minor processes may lead to a deviation from the theoretical 1:1 molar ratio of $\mathrm{CO}_{2}$ and ethanol.

$$
\mathrm{C}_{6} \mathrm{H}_{12} \mathrm{O}_{6}=2 \mathrm{C}_{2} \mathrm{H}_{5} \mathrm{OH}+2 \mathrm{CO}_{2}
$$


This basic natural process has been utilized by the mankind for many thousands of years mostly in food related industries, such as winery, brewery, and bakery (JOHNSON \& ECHAVARRIERASUN, 2011). New application area for ethanol is in the transport sector, where depending on the origin of the glucose, there are basically two technologies. In case the six carbon sugar originates from starch (mostly wheat or corn grain) or sugar (mostly sugar beet or sugar cane) materials the technology is called first generation bioethanol production, which can be considered as a mature technology (SANCHEZ \& CARDONA, 2008). Second generation bioethanol production utilizes lignocellulose as feedstock in form of agricultural/forestry residues (like corn stover, wheat straw, sweet sorghum bagasse) or dedicated energy crops. The utilization of byproducts makes second generation technology advantageous over first generation where the feedstock portfolio may compete with food uses. However, a lot of research has been done so far, due to the complex, multi-steps pattern of lignocellulose conversion (Yulin \& Mosier, 2008), the process requires further research.

This research and development needs dedicated, easy-to-use and reliable devices to monitor ethanol fermentation in a direct and comparable manner. During ethanol fermentation concentrations of both substrates and products can provide important information about the state of the process. However, because of the above-mentioned minor glucose conversion pathways, differences may occur in tracking either the amount of the substrate or the products. To follow fermentations, basically three methods can be used.

The first and most common one is sampling, when concentrations of both substrates and products can be measured from the fermentation broth after withdrawal of the sample. The results are usually obtained after time-consuming analyses when the actual state of the system has already changed, thus it is not possible to deliver information about the system immediately. Moreover, with the regular sampling the opening of the system is inevitable, which can cause infection and changes in the environmental parameters ( $\mathrm{pH}$, temperature).

The second possibility is to measure the solved substances by an online monitoring method where the selective detector is part of the fermentation system. Biosensors with enzymatic detection are able to measure glucose and/or ethanol from the reactor directly, however, while these biosensors possess good sensitivity and stability, they usually have poor selectivity (LEI et al., 2006). Biosensors can be used in fermentors but may not be applicable in small flask reactors where several experiments are run in parallel.

Numerous ways are known - including volume displacement devices, lubricated syringes, manometer-assisted syringes, calibrated pressure manometers and automatic respirometers - to measure the produced $\mathrm{CO}_{2}$ that leaves the reactor, as being the third method (Young et al., 1991). The main advantage of measuring the effluent gas is that there is no need to open up the fermentation vessel and also the continuous formation of the gas makes the real time monitoring possible.

However, gas volume measurement is not selective to a compound, the process tracked this way must be well established (such as ethanol fermentation or biogas formation). Ethanol production can be estimated from the volume of $\mathrm{CO}_{2}$ produced based on equation 1 with the respective assumptions and realizing the limits of this equation describing biological systems. Therefore, these equipments measuring the gas formed may not deliver exact results by means of ethanol determination (since the real connection between gas produced and ethanol is not easy to establish), but make it possible to track the fermentation.

The most sophisticated method to measure the amount of gas is the principle of displacement (i.e. conventional Mariotte bottle): the gas is trapped in a fluid sealed device where same volume of fluid is "displaced" by the effluent gas leading to a signal in a sensor. 
Different solutions have been proved to make this basic principle work in a continually functioning design, when the gas is allowed to leave the system to the atmosphere, and the device can return to the starting position without (VeIGA et al., 1990) or with integration of pumps and/or valves (Macías et al., 1995; Sмiтh \& STÖcKLE, 2008).

Regardless of the exact type of the trapping device, many models have been proposed to monitor biological gas formation processes, in most cases related to the biogas production. The signal in the most cases is the change in the level of the trapping fluid and many sensors are available to sense this change. For instance, sensing the difference between the gas and the fluid by means of electrical conductivity (Veiga et al., 1990; MACías et al., 1995) or different optical properties (LiU et al., 2004) have also been reported to work effectively in monitoring. In case the device is not continuously opened to atmosphere, the pressure of the trapped gas can be used as signal as well. As an example, pressure transducers in combination with valves and a ballast chamber can be used; the pressure is building up until a set pressure activates the valve, releasing the gas into the atmosphere (Guwy et al., 1995). Other option is that the gas lifts the counter-weighted, floating cap, which triggers the sensor activating a timer to run a pump removing the built-up pressure and restarting the cycle (SMITH \& STÖcKLE, 2008).

Taking into the account the advantages of real time effluent gas monitoring, the aim of our study was to develop and statistically evaluate an online monitoring system for ethanol fermentation, which is able to track the fermentation in real time, delivering an immediate feedback of the process. Moreover, as monitoring is based on the simple method of measuring effluent gas, other processes with gas formation (e.g. biogas production) can be tracked as well.

\section{Materials and methods}

\subsection{Raw materials and strains}

Synthetic medium containing glucose $\left(10 \mathrm{~g} \mathrm{l}^{-1}, 20 \mathrm{~g} \mathrm{l}^{-1}, 30 \mathrm{~g} \mathrm{l}^{-1}, 40 \mathrm{~g} \mathrm{l}^{-1}, 60 \mathrm{~g} \mathrm{l}^{-1}\right.$, and $\left.80 \mathrm{~g} \mathrm{l}^{-1}\right)$ and the salts $\left(0.3 \mathrm{~g} \mathrm{l}^{-1} \mathrm{MgSO}_{4}, 2.0 \mathrm{~g} \mathrm{l}^{-1} \mathrm{NH}_{4} \mathrm{Cl}\right.$, and $\left.1.0 \mathrm{~g} \mathrm{l}^{-1} \mathrm{~K}_{2} \mathrm{HSO}_{4}\right)$ were used for ethanol fermentation experiments by baker's yeast. For fermentation experiments fresh blocks of baker's yeast (Saccharomyces cerevisiae), product of Lesaffre Magyarország Élesztőgyártó és Kereskedelmi Kft., Budafok, Hungary were purchased in a local store on the day of use.

\subsection{Fermentation}

Batch fermentations of $200 \mathrm{ml}$ synthetic media were carried out at $30{ }^{\circ} \mathrm{C}$ at $\mathrm{pH} 4.8$ with the addition of $2 \mathrm{~g} \mathrm{l}^{-1}$ dry yeast in $250 \mathrm{ml}$ screw-capped bottles with magnetic stirring (250 r.p.m.) connected to the monitoring device. The fermentation was tracked by measuring the $\mathrm{CO}_{2}$ production by a novel online fermentation module device developed by Nonfood group (at Budapest University of Technology and Economics, Department of Applied Biotechnology and Food Science) and Stereo Vision Ltd. In order to correctly carry out the statistical evaluation of the device, fermentations were performed at least in duplicates in parallel and also with repetition at other times.

At the end of the fermentations, when the $\mathrm{CO}_{2}$ production ceased, flasks were sampled. Samples were centrifuged in $50 \mathrm{ml}$ centrifuge tubes at 9000 r.p.m. for 5 min. Supernatants were analysed for ethanol concentration by HPLC in order to establish a connection between the produced gas volume and the amount of ethanol. 


\subsection{Analytical methods}

Samples for HPLC analysis were prepared by filtering through a regenerated cellulose syringe filter with $0.45 \mu \mathrm{m}$ pore size (ProFill, Langerwehe, Germany). The residual sugar and ethanol contents of the fermented samples were separated on an Aminex ion exclusion HPX$87 \mathrm{H}$ cation-exchange column (BioRad, Hercules, CA, USA) running at $65^{\circ} \mathrm{C}$ with $5 \mathrm{mM}$ sulphuric acid as mobile phase at a flow rate of $0.5 \mathrm{ml} \mathrm{min}{ }^{-1}$. After separation, the compounds were detected by a Shimadzu RID-I0A refractive index detector (Shimadzu, Kyoto, Japan).

\subsection{Evaluation of the results}

Both the calibration of the device and test measurements with the synthetic media were statistically evaluated. Raw experimental data (number of displacement events and recorded gas volumes) were processed with STATISTICA 9.0 software (StatSoft, Inc., Tulsa, OK, USA) in order to establish the reliability of the calibration method and the device itself.

The calibration method was verified by $t$-tests for independent samples repeated on different days and outliers for each channel were identified with Grubbs test by comparing the calculated minimum and maximum $\mathrm{Z}$ values to a critical one derived from statistical database. The null hypothesis of the test is that there is no outlying observation. When the calculated $\mathrm{Z}$ value is below the critical value, the null hypothesis is accepted. The calculation for Grubbs tests is based on equation 2:

$$
\mathrm{Z}=\left|\frac{\mathrm{x}_{\mathrm{q}}-\overline{\mathrm{x}}}{\mathrm{s}}\right|
$$

where $\mathrm{Z}$ is the Grubbs test statistic, $\mathrm{x}_{\mathrm{q}}$ is the measurement's minimum or maximum value (displacement event volume on each channel), $\bar{x}$ is the average of the displacement event volumes on each channel, $\mathrm{s}$ is the standard deviation.

The significance level for $t$-test for independent samples was set to 0.05 . The equality of variances in the two compared distributions - the prerequisite of the $t$-tests - was also automatically tested by F-tests each time by the software (data not shown).

The results of test runs (recorded gas volumes) on the synthetic media were analyed by analysis of covariance (ANCOVA) with the factors of day, channel and glucose concentration. Prerequisites of ANCOVA were also tested. The purpose of ANCOVA was to calculate the impact between days and channels expressed as deviations, as well as the total deviation.

Furthermore, determination of ethanol concentration with online fermentation monitoring system was also attempted based on the produced amount of $\mathrm{CO}_{2}$, which according to equation 1 is stoichiometrically equal to the amount of ethanol. Since this equation does not consider many factors arising from a biological system (molar ratio can differ, $\mathrm{CO}_{2}$ can come from other sources) it may not be appropriate for statistical analyses and exact calculations. Nevertheless, equation 3 based on the ideal gas law tries to establish a connection between the valuable (ethanol) and measured $\left(\mathrm{CO}_{2}\right)$ products not taking into account the amount of substrate (hence not relying on conversion values). 


$$
\mathrm{c}_{\mathrm{EtOH}}\left[\mathrm{g} \mathrm{l}^{-1}\right]=\frac{\left(\mathrm{p}_{\mathrm{air}}[\mathrm{Pa}]+0.09 \times 1000 \times 9.81\right) \times \mathrm{V}_{\mathrm{CO}_{2}}[\mathrm{ml}] \times 10^{-6}}{\mathrm{R} \times \mathrm{T}_{\text {air }}[\mathrm{K}]} \times \frac{46}{\mathrm{~V}_{\text {sample }}[1]}
$$

Where $\mathrm{p}_{\text {air }}$ is the average atmospheric pressure during the measurement [Pa], 0.09 is the maximum deflection in the U-tube just before inversion [m], 1000 is the density of $10^{-2} \mathrm{M}$ $\mathrm{HCl}$ which was applied to minimize $\mathrm{CO}_{2}$ loss by solution $\left[\mathrm{kg} \mathrm{m}^{-3}\right], 9.81$ is the acceleration of gravity $\left[\mathrm{m} \mathrm{s}^{-2}\right], \mathrm{V}_{\mathrm{CO} 2}$ is the total volume of the produced gas $[\mathrm{ml}], 10^{-6}$ is a factor to convert $\mathrm{ml}$ to $\mathrm{m}^{3}, \mathrm{R}$ is the Regnault constant $\left[8.314 \mathrm{~J} \mathrm{~mol}^{-1} \mathrm{~K}^{-1}\right], \mathrm{T}_{\text {air }}$ is the average air temperature during the measurement $[\mathrm{K}], 46$ is the molar weight of ethanol $\left[\mathrm{g} \mathrm{mol}^{-1}\right], \mathrm{V}_{\text {sample }}$ is the volume of the sample [1].

Simplification can be made in equation 3 using standard conditions $\left(25^{\circ} \mathrm{C}, 100000 \mathrm{~Pa}\right)$ that well approximates actual laboratory conditions during the study (Eq. 4).

$$
\mathrm{c}_{\mathrm{EtOH}}\left[\mathrm{g} \mathrm{l}^{-1}\right]=\frac{0.0019 \times \mathrm{V}_{\mathrm{CO}_{2}}[\mathrm{ml}]}{\mathrm{V}_{\text {sample }}[1]}
$$

It was assumed that the changes of the neglected parameters are in a certain interval during the measurements (supported by observations) and also that this can be considered as error of the parameter. In order to estimate the error due to this simplification error propagation calculation was tested with possible maximal variances from the standard conditions under the conditions of the experiments (Eq. 5).

$$
\sigma_{\mathrm{EtOH}}^{2}=\left(\frac{\partial \mathrm{c}_{\mathrm{EtOH}}}{\partial \mathrm{P}_{\mathrm{a}}}\right)^{2} \times \sigma_{\mathrm{P}_{\mathrm{a}}}^{2}+\left(\frac{\partial \mathrm{c}_{\mathrm{EtOH}}}{\partial \mathrm{T}_{\mathrm{a}}}\right)^{2} \times \sigma_{\mathrm{T}_{\mathrm{a}}}^{2}+\left(\frac{\partial \mathrm{c}_{\mathrm{EtOH}}}{\partial \mathrm{m}}\right)^{2} \times \sigma_{\mathrm{m}}^{2}
$$

Where $\sigma^{2}$ is the variance, first term $\left(\mathrm{P}_{\mathrm{a}}\right)$ describes the maximal variance of the standard air pressure during the measurement based on data of the Hungarian Meteorological Service $[15 \mathrm{hPa}]$, second $\left(\mathrm{T}_{\mathrm{a}}\right)$ the variance of the standard air temperature during the measurement $[3 \mathrm{~K}]$, while third one $(\mathrm{m})$ the variance derived from the error of the scales (mass of feedstock in each fermentor) $[0.01 \mathrm{~g}], \mathrm{c}_{\mathrm{EtOH}}$ is the concentration of ethanol $\left[\mathrm{g} \mathrm{l}^{-1}\right]$.

The result of the calculation is \pm 0.0052 , which is minor so the simplification of equation 3 is valid and does not result in error propagation.

\section{Results and discussion}

\subsection{Description and operation principle}

In order to evaluate and draw correct conclusions from experiments, proper methods and dedicated devices are necessary. First, a device was developed that is capable of monitoring ethanol fermentation in real time. With this feature and the eight parallel sample sites, it is possible to see the differences between substrates, pretreatments and media already during fermentation and not only after sampling and timely analyses.

The system is basically divided into two parts, the measuring device and the processing software. The main component of the device, where the effluent $\mathrm{CO}_{2}$ is trapped for an interval, 
is a U-tube filled with $10^{-2} \mathrm{M} \mathrm{HCl}$ solution to prevent $\mathrm{CO}_{2}$ loss and equipped with two copper electrodes of different lengths. The end of the longer electrode permanently immerses in the acid solution, while the shorter one is only in the liquid when the device under pressure, i.e. effluent gas enters the tube.

The U-tube has a $15 \mathrm{~mm}$ internal diameter, and a height of about $25 \mathrm{~cm}$. Top of the left leg is closed, and the gas inlet tube from the fermentor is also connected here. The right leg is open to the atmosphere and the electrodes are also placed here. Between the two legs a transfer tube is placed with $3 \mathrm{~mm}$ internal diameter and connection points to the main legs at different heights (Fig. 1).

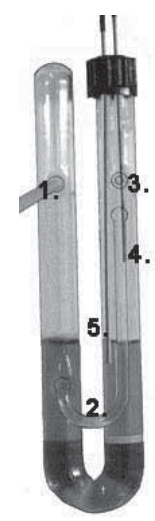

Fig. 1. Structure of the U-tube used to measure the amount of produced gas as part of the online fermentation monitoring system. Legend: 1 . Gas introductory tube (from reactor); 2. transfer tube between the two legs; 3. gas outlet; 4 . shorter electrode ( $+4.4 \mathrm{~V}$ voltage $)$; 5 . longer electrode $(0 \mathrm{~V}$ voltage $)$

This structure of the U-tube and thus the operation principle were originally described by VeIGA and co-workers (1990) in order to measure the volume of biogas in aerobic digestion. Compared to that design some minor differences were applied to the U-tube used in our setup. First, the size was decreased resulting in displacement events with volume around 12 $\mathrm{ml}$ instead of the $50 \mathrm{ml}$ as described by VEIGA and co-workers (1990). Second, the original design was supplied by a voltage of $220 \mathrm{~V}$, while in our case it was only $4.4 \mathrm{~V}$ supplied trough the USB port of a PC. Nevertheless, the major difference is the method of signal processing: with being connected to a PC, it became possible to follow the process in real time and obtain a view into the reaction kinetics. This real time tracking is possible on 8 channels simultaneously - to measure more samples at the same time is not mentioned by VeIGA and co-workers (1990). Furthermore, we carried out a statistical evaluation and attempted to define the accuracy of our device specific for ethanol fermentation.

The operation principle is the following: the produced $\mathrm{CO}_{2}$ enters in either leg of the tube and leaves through the other while the same volume of liquid as its own is moving through the legs. The gas leaves the tube in fixed increments through an inverted siphon (transfer tube) placed between the two legs of the U-tube. With this design the application of pumps and/or valves is also avoided. The different lengths of the electrodes and thus their changing contact with the solution depending on the level make possible to detect changes in the liquid surface level by means of conductivity and thereby count the number of displacement events. These events get registered on a personal computer by a software developed by Stereo Vision Ltd. As the measuring device was calibrated (in order to know the volume of one 
displacement event, $\mathrm{ml}$ event $^{-1}$ ), the exact volume leaving the tube during a displacement event is known, so by cumulating the number of these events the total volume can be calculated. As the main feature, the software displays a cumulated $\mathrm{CO}_{2}$ versus time plot in real time. The measured data and the plot can be exported to a Microsoft Excel file for further editing and studying.

\subsection{Principle and statistical verification of calibration}

As the volume of a displacement event (event volume) is of crucial importance for reliable results, the reliance and repetitiveness of the calibration method were investigated based on obtained experimental data. For this reason calibration on each channel was repeated many times; even at different days (meaning a total of 48 measurements, 6 per channel, mean values shown in Table 1). The null hypothesis of the testing was that the same event volumes got obtained.

The principle of the calibration is also displacement, using an empty bottle instead of the fermentation reactor connected to the device. This bottle is used to accept the water of known volume running through the system and displacing the same amount of air. The 8 channels were parallel calibrated with $500 \mathrm{ml}$, approximately $31{ }^{\circ} \mathrm{C}$ (the temperature of fermentation) water that displaces the same volume of air leaving the bottle via the U-tube imitating displacing events. The displacement event volume $\left(\mathrm{ml}\right.$ event $\left.{ }^{-1}\right)$ was calculated by dividing the volume of the transferred water (measured with a measuring cylinder after the calibration) with the number of displacement events counted by the software.

Repetitions were made on two different days $(3+3)$ on the same channel. The averages of each day (day A versus day B) were compared by $t$-tests hypothesizing no effect of date on obtained displacement event volumes. In each case, the F-tests prior to the $t$-test gave acceptable results. As shown by $t$-tests (in all cases $\mathrm{P}>0.05$ ), the difference between days in case of calibrating the same channel is not significant (Table 1), meaning that the date (and thus possible changes of conditions) of calibration is not relevant in obtaining the displacement event volume of a channel.

In case of all channels the ranges of displacement event volumes were inside a range of $2 \mathrm{ml}$. Furthermore, during the calibration no outliers and extremes were obtained, showing that the calibration is restricted to a range regardless of its day and channel. The lack of extreme values and outliers was also confirmed for each channel by Grubbs test using the raw data that based for the mean values in Table 1 (meaning 6 points per channel - critical value for $\mathrm{Z}$ at 6 samples is 1.89).

In case of all channels, the average displacement event volumes were about $12 \mathrm{ml}$, that is - assuming the estimation based on equation 4 - around a resolution of $0.023 \mathrm{~g}$ ethanol, presenting a method with good sensitivity. It should be noted that the volumes cannot be compared among channels, since the U-tubes are hand-made and the level (thus the amount) of $\mathrm{HCl}$ solution is different. Therefore, the variation of volumes is normal and thus the calibration can be verified only for a given channel not among the channels.

Based on the repeated calibration and Grubbs tests, the method of calibration was accepted, as well as the obtained displacement event volumes were considered as reliable and accurate. The displacement event volumes in Table 1 were used through the fermentation tests to calculate volumes of counted displacement events (the conversion process is carried out by the software delivering real time gas volume plots). 
Table 1. Evaluation of calibration displacement events of the online fermentation monitoring system

\begin{tabular}{|c|c|c|c|c|}
\hline \multirow{2}{*}{ Channel } & $\begin{array}{l}\text { Average volume of } \\
\text { displacement event }\end{array}$ & Standard deviation & \multirow{2}{*}{ Number of repetitions } & \multirow{2}{*}{$\begin{array}{l}t \text {-value } \\
(\mathrm{P}=0.05)\end{array}$} \\
\hline & \multicolumn{2}{|c|}{$(\mathrm{ml})$} & & \\
\hline 1 & 11.24 & 0.08 & 6 & \\
\hline 2 & 11.50 & 0.17 & 6 & \\
\hline 3 & 11.45 & 0.48 & $3+3$ & \\
\hline 3 (day A) & 11.75 & 0.36 & 3 & 0.077 \\
\hline 3 (day B) & 11.03 & 0.06 & 3 & \\
\hline 4 & 12.22 & 0.38 & $3+3$ & \\
\hline 4 (day A) & 12.14 & 0.26 & 3 & 0.826 \\
\hline 4 (day B) & 12.21 & 0.50 & 3 & \\
\hline 5 & 11.95 & 0.23 & 6 & \\
\hline 6 & 12.09 & 0.28 & $3+3$ & \\
\hline 6 (day A) & 12.36 & 0.25 & 3 & 0.137 \\
\hline 6 (day B) & 11.83 & 0.40 & 3 & \\
\hline 7 & 11.66 & 0.31 & $3+3$ & \\
\hline 7 (day A) & 11.67 & 0.33 & 3 & 0.909 \\
\hline 7 (day B) & 11.64 & 0.40 & 3 & \\
\hline 8 & 11.97 & 0.22 & 6 & \\
\hline
\end{tabular}

\subsection{Verification of the device}

Applying the verified event volumes for each channel, the device was used to track real fermentations running on synthetic media. Similar to calibration, the fermentation runs were repeated many times on different days (marked by capital Latin letters) and also same samples were run on different channels (marked by Roman numbers) in order to verify the compatibility of channels (Table 2).

Table 2. Experimental matrix of test runs on different media, glucose concentration is indicated in $\mathrm{g}^{-1}$

\begin{tabular}{lllllll}
\hline Day & A & B & C & D & E & F \\
\hline 1 & 20 & 40 & 60 & 20 & 60 & 30 \\
2 & 20 & 40 & 60 & 20 & 60 & 30 \\
3 & 20 & 40 & 60 & 20 & 60 & 30 \\
4 & 20 & 40 & 60 & 40 & 60 & 30 \\
5 & 40 & 20 & 80 & 40 & 80 & 10 \\
6 & 40 & 20 & 80 & 40 & 80 & 10 \\
7 & 40 & 20 & 80 & 60 & 80 & 10 \\
8 & 40 & 20 & 80 & 60 & n.d. & 10 \\
\hline
\end{tabular}

nd: not determined 
Based on the above experimental matrix a total of 47 useful fermentation results (registered displacement event/gas volume) were obtained on different glucose concentrations (Table 3). These 47 experimental data were the input for the statistical verification of the device.

Table 3. Results of the 47 fermentation test runs on different synthetic media

\begin{tabular}{lccccc}
\hline $\begin{array}{l}\text { Glucose } \\
\text { concentration } \\
\left(\mathrm{g}^{-1}\right)\end{array}$ & $\begin{array}{c}\text { Total number of } \\
\text { runs }\end{array}$ & $\begin{array}{c}\text { Interval of } \\
\text { recorded } \\
\text { displacement } \\
\text { events }\end{array}$ & $\begin{array}{c}\text { Interval of } \\
\text { recorded gas } \\
\text { volume }(\mathrm{ml})\end{array}$ & $\begin{array}{c}\text { Mean value of } \\
\text { recorded gas } \\
\text { volume }(\mathrm{ml})\end{array}$ & $\begin{array}{c}\text { Standard } \\
\text { deviation, ml } \\
(\%)\end{array}$ \\
\hline 10 & 4 & $35-38$ & $419.0-447.3$ & 434.9 & $12.9(3.0)$ \\
20 & 11 & $80-98$ & $956.0-1116.2$ & 1036.6 & $46.6(4.5)$ \\
30 & 4 & $136-156$ & $1655.1-1776.8$ & 1695.3 & $71.6(4.2)$ \\
40 & 11 & $178-204$ & $2127.1-2369.6$ & 2263.9 & $86.2(3.8)$ \\
60 & 10 & $268-328$ & $3079.8-3735.9$ & 3451.7 & $192.3(5.6)$ \\
80 & 7 & $254-350$ & $3040.4-4081.0$ & 3608.1 & $463.3(12.8)$ \\
\hline
\end{tabular}

a Displacement event multiplied by displacement event volumes (specific for each channel), as determined by in chapter 2.2, therefore the channel with the less event number may be not equal with the channel resulting the less volume

The results of the tracking are the real time cumulated gas volume plots (Fig. 2). As these plots are available during the whole fermentation in real time on the display, it is easy to follow the process and see immediately the actual state of the fermentation, as well as direct comparison between the 8 channels with different experimental designs is also possible.

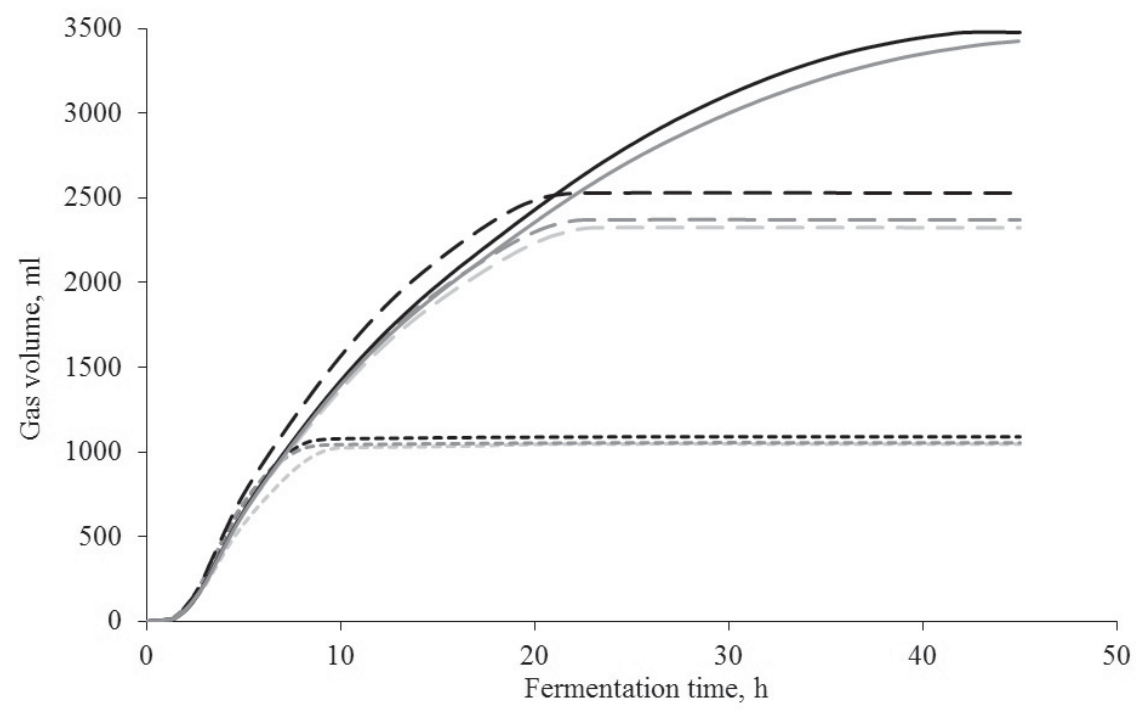

Fig. 2. Primary results delivered by the online fermentation monitoring system: real time gas volume plots on different channels and glucose amount per reactor: - - : channel 1: $4 \mathrm{~g}$ glucose; - - - - : channel 2: $4 \mathrm{~g}$ glucose; - - - - : channel 3: $4 \mathrm{~g}$ glucose; — — : channel 4: $8 \mathrm{~g}$ glucose; — — : channel 5: $8 \mathrm{~g}$ glucose; — - channel 6: $8 \mathrm{~g}$ glucose; — : channel 7: $12 \mathrm{~g}$ glucose; — : channel 8: $12 \mathrm{~g}$ glucose 
Based on this plot, the device is also suitable for monitoring the kinetics of fermentation. For instance in the current case, it can be observed that fermentation kinetics do not depend on the concentration of glucose, since each curve has the same profile (Fig. 2). In the initial phase, the volume of the produced gas is proportional with the glucose concentration. The curves flatten before reaching the plateau of which time point depends on the amount of sugar. As an advantage of the real time tracking, the end of fermentation can be recognized immediately once the plateau is reached, meaning that gas formation had stopped.

The compatibility of the final results ( 47 recorded final gas volumes - plateaus of the 47 time-volume plots) was examined by ANCOVA. The factors of ANCOVA were the different days and channels on which the actual result was obtained. The different glucose concentrations were set as fixed factor. Firstly, the eligibility criteria for ANCOVA were also tested. The results proved that the variations of obtained gas volumes (represented by residuals) follow normal distribution (normal probability plot). The homogeneity of variances assessed by Cochran test (among the samples run on different glucose concentrations) is also accepted. However, because of some microbial phenomenon (also referred at equations 1 and 3 but not incorporated in them), baker's yeast consumed glucose in $80 \mathrm{~g} \mathrm{l}^{-1}$ (16 g per sample) with very poor efficiency resulting in a nonlinear relationship between the amount of glucose and produced gas volume. This discrepancy is also reflected in the high standard deviation as shown in Table 3 in cases of fermentations run on $16 \mathrm{~g}$ glucose. When removing the results obtained on $16 \mathrm{~g}$ glucose (only including results of $12 \mathrm{~g}$ and below) linear connection was established, thus only these results (a total of 40 recorded final gas volume) were used for ANCOVA.

As a result of ANCOVA, standard deviations of $58.8 \mathrm{ml}$ and $82.1 \mathrm{ml}$ were calculated representing the impacts of channel and date of tests, respectively. Based on this a combined standard deviation including all effects of $116.2 \mathrm{ml}$ was calculated, which is approximately equal to 10 displacement events (exact value depends on the channel) or - assuming the estimation based on equation 4 - around $0.23 \mathrm{~g}$ of ethanol (if there is a discrepancy from the theoretical equation this deviation is still not significant). In view of these results it can be stated that the choice of channel does not influence the measurement; thus all channels are compatible with the others and channels can reliably repeat measurements. It is also worth to note that the statistical evaluation by ANCOVA did not consider any biological effect and changes but they cannot be excluded. Hence, the calculated standard deviations may be not exclusively derived from the device and other measurement related issues but also perhaps from biological properties of the system not taken into account.

\subsection{Verification of the amount of ethanol formed}

At the end of fermentations, the concentration of ethanol in each sample was determined by HPLC technique and plotted against the gas volumes recorded (Fig. 3). Based on this a slope of 0.00158 was obtained ( $1 \mathrm{ml}$ of $\mathrm{CO}_{2}$ per $0.00158 \mathrm{~g}$ ethanol). This connection is displayed together with the solely theoretical equation 4 (Fig. 3) for comparison.

The ratio of calculated ethanol amount and the HPLC measured ethanol amount were compared. In case of samples run on $2 \mathrm{~g}$ glucose, this ratio was above 1 showing that in these cases a higher ethanol concentration was calculated based on the gas volume. In case of larger glucose amounts the ratios were in almost all cases below 1 but above 0.8 . Taking all samples into account an average ratio of 0.90 was obtained, meaning that the differences between HPLC results and calculated ethanol values were in a $10 \%$ range. This presents well that the theoretical equation can deliver a good and instant preliminary estimate that, however, 
cannot replace exact determinations. The reason for this discrepancy may be due to biological properties of the system, some possible errors related to the device or any other conditions outside the device not taken into account.

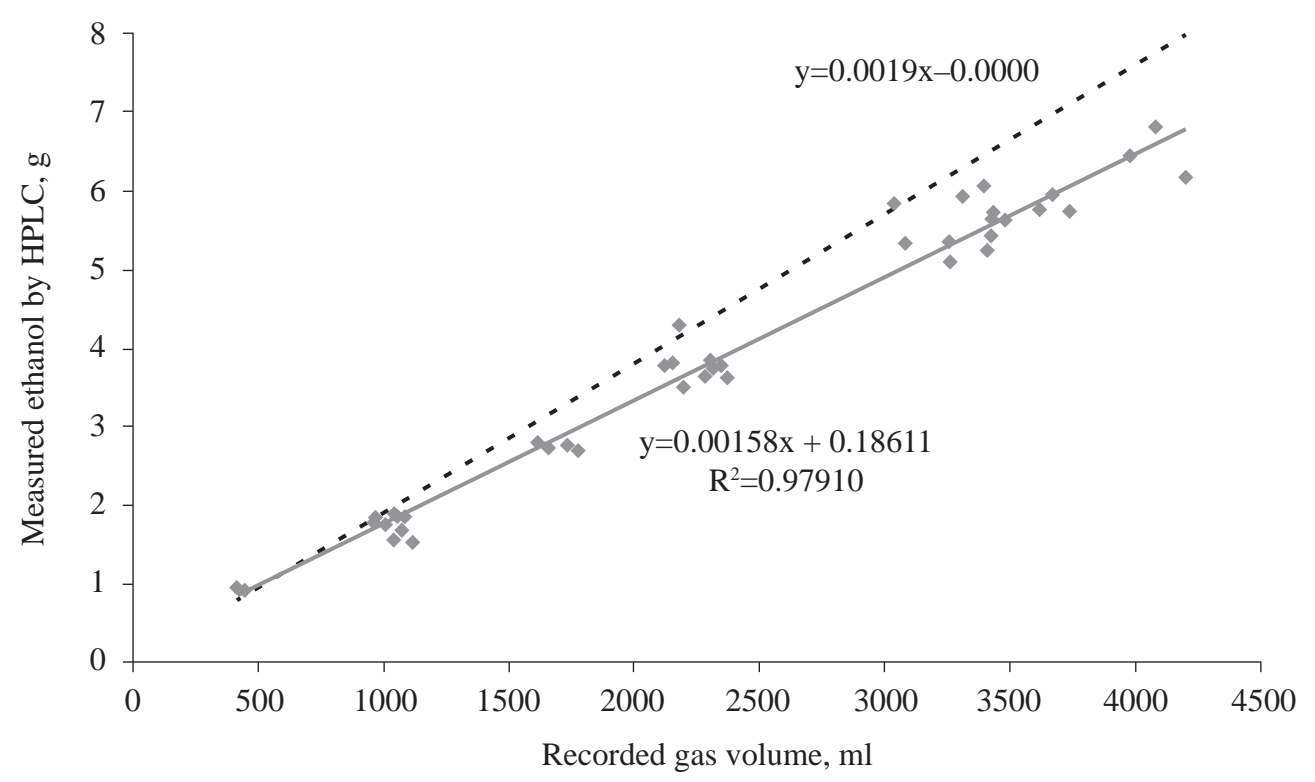

Fig. 3. Measured ethanol by HPLC plotted against recorded gas volume and its linear fit, as well as the hypothetical relationship according to equation 4 is added

\section{Conclusions}

A device and software were developed that is suitable to follow ethanol fermentation or any other process with gas formation in real time and on parallel channels. Advantage of this approach is the immediate results and the possibility to study the kinetics and the impact of different experimental designs. Hereby, this device and its calibration were statistically evaluated based on experimental data. The results show that the calibration method and the so obtained displacement event volumes are reliable, such as the tracking of test fermentations proved to deliver results with acceptable standard deviations as found by ANCOVA. Setting up an equation to estimate ethanol concentration from the gas volume produced was also attempted, however, a discrepancy was observed between this theoretical equation and the one fitted on the obtained ethanol concentration by HPLC. Despite this discrepancy the theoretical equation can be considered applicable to estimate ethanol concentration immediately at any time during fermentations. Nevertheless, taking into account the biological processes and possibilities that can occur in a fermentation broth, it cannot be stated that this discrepancy is because of an error in the device. Consequently, as shown by statistical tests, the device is suitable for monitoring ethanol fermentation due to the advantages of real time parallel tracking of samples. 
J. GeIER (Stereo Vision Ltd.) is gratefully acknowledged for the assistance in developing the device and for his valuable comments on drafting the manuscript. S. KEMÉNY (Budapest University of Technology and Economy) is also gratefully acknowledged for his assistance in the statistical evaluation.

\section{References}

Guwy, A.J., HAwKeS, D.L.\& HAwKes, F.R. (1995): On-line low flow high-precision gas metering systems. Water Research, 29, 977-979.

Johnson, E.A. \& Echavarri-Erasun, C. (2011): Yeast biotechnology. -in: Kurtzman, C.P., Fell, J.W. \& Boekhout, T. (Eds) The yeasts. A taxonomic study ( $5^{\text {th }}$ ed.), Elsevier BV., New York, pp. 21-44.

Lei, Y., Chen, W. \& Ashoк, M. (2006): Microbial biosensors. Anal. Chim. Acta, 568, 200-210.

Liv, J., Olsson, G. \& Mattiasson, B. (2004): A volumetric meter for monitoring of low gas flow rate from laboratoryscale biogas reactors. Sensors Actuators B, 97, 369-372.

Macías, M., Pérez, M., Caro, I. \& Cantero, D. (1995): Automatic gas meter for laboratory ferments. Biotechnol. Techniques, 9, 655-658.

Russell, I. (2003): Understanding yeast fundamentals. -in: Jacques, K.A., Lyons, T.P. \& Kelsall, D.R. (Eds) The alcohol textbook. A reference for the beverage, fuel and industrial alcohol industries ( $4^{\text {th }}$ ed.), Nottingham University Press, Nottingham, UK, pp. 85-120.

SAncheZ, O.J. \& CARDona, C.A. (2008): Trends in biotechnological production of fuel ethanol from different feedstock. Bioresource Technol., 99, 5270-5295.

Sмith, S.A.\&Stöckle, C.O. (2008): A biogas meter with adjustable resolution and minimal back-pressure. Bioresource Technol., 99, 8537-8539.

Veiga, M.C., Soto, M., Méndez, R.\&Lema, J.M. (1990): A new device for measurement and control of gas production by bench scale anaerobic digesters. Water Res., 24, 1551-1554.

YounG, J.C., Kuss, L.M. \& Nelson, A.M. (1991): Use of anaerobic respirometers for measuring gas production in toxicity and treatability tests. Proceedings of the 84th Annual Meeting of the Air and Waste Management Association. Vancouver BC, Canada.

Yulin, L.\&Mosier, N.S. (2008): Current technologies for fuel ethanol production from lignocellulosic plant biomass. -in: VermerRis, W. (Ed.) Genetic improvement of bioenergy crops. Springer Science + Business Media, LLC, New York, NY, USA, pp. 161-182. 